\title{
Tracking Error:
}

Ex-Ante versus Ex-Post Measures

Soosung Hwang and Steve Satchell 


\title{
Tracking Error: Ex-Ante versus Ex-Post Measures
}

\author{
Soosung Hwang \\ Department of Banking and Finance, \\ City University Business School, UK \\ and \\ Stephen E. Satchell \\ Faculty of Economics and Politics and Trinity College, \\ Cambridge University, UK
}

\begin{abstract}
In this paper we show that ex-ante and ex-post tracking errors must necessarily differ, since portfolio weights are ex-post stochastic in nature. In particular, ex-post tracking error is always larger than ex-ante tracking error. Our results imply that fund managers always have a higher ex-post tracking error than their planned tracking error, and thus unless our results are considered, any performance fee based on ex-post tracking error is unfavourable to fund managers.
\end{abstract}

Key Words: Tracking Error, Risk, and Performance Measurement, Active Management.

JEL Code:

* Corresponding Author. Faculty of Economics and Politics, Austin Robinson Building, Sidgwick Avenue, Cambridge CB3 9DD, UK, Tel: 44 (0)1223 335213, Fax: 44 (0)1223 335475. 


\section{Introduction}

Portfolio performance is usually evaluated against a prespecified benchmark portfolio. One most frequently used measure is Tracking Error (TE), sometimes defined as differences between portfolio returns and the benchmark portfolio returns. TE is simple and easy to calculate as well as a powerful tool in structuring and managing index funds. Two common sources of tracking errors come from the attempts to outperform the benchmark and the passive portfolio replication of the benchmark by a sampled portfolio.

In the analysis of TE, outperforming the benchmark is equivalent to having a positive expected TE; we call the mean TE 'expected relative return' in this study. The risk related to TE is measured by the volatility of the difference between managed portfolio returns and benchmark returns. The volatility is called TE throughout our study. ${ }^{1}$ Thus, minimising TE as well as maximising expected relative return is a sensible goal for investors.

Most studies on TE have concentrated on how to minimise TE, or how to maximise expected relative return for a given TE; see Larsen and Resnick (1998), and Baierl and Chen (2000). Roll (1992) derived an efficient portfolio in 'TE - expected relative return' space and showed that a Markowitz efficient frontier dominates the efficient frontier derived with TE.

Pope and Yadav (1994), on the other hand, showed that serial correlation of the returns differences between an index fund portfolio and the underlying benchmark portfolio results in a biased estimate of TE. For example, the annual TE calculated with the daily TE will not be a good estimate of the true annual TE in the presence of serial correlation.

In this paper, we suggest a different source of bias in the TE, which arises from the stochastic nature of portfolio weights. We compare two measures of TE, ex-ante and ex-post, and show that the bias comes from the unconditionally stochastic nature of portfolio weights. That is, since portfolio weights are themselves random variables, there is additional variation ex-post not accounted for ex-ante. Therefore, the bias can only be found in active portfolios. However, we show that it will be also found in

\footnotetext{
${ }^{1}$ Tracking error is defined in different ways in different studies. For example, Pope and Yadav (1994), Lee (1998), and Rudolf, Wolter and Zimmermann (1999) defined tracking error as variance (standard deviation) of the difference between portfolio returns and benchmark returns. On the other hand, Clarke, Krase, and Statman (1994), Roll (1992) defined tracking error as difference between portfolio returns and the benchmark portfolio returns. In this study we follow the definition of the former since it is widely accepted by practitioners.
} 
passive portfolios whose portfolio weights are not stochastic due to rebalancings.

We use two different measures for TE; one is the variance (standard deviation) of the returns difference between portfolio and the benchmark portfolio, and the other is the mean absolute deviations (MAD) of them. TE measured with standard deviation (variance) will be denoted as $T E_{S D}$ (for variance, $T E_{S D}^{2}$ ) whilst TE measured with the MAD will be represented as $T E_{M A D}$. We show that when the difference between portfolio weights and the benchmark portfolio weights is stochastic, ex-ante $T E_{S D}\left(T E_{S D}^{2}\right)$ is necessarily downward biased. The results in this study imply that the realised $T E_{S D}$ is always larger than the planned $T E_{S D}$. On the other hand, we cannot conclude if ex-ante $T E_{M A D}$ is downward or upward biased in the presence of stochastic difference between portfolio weights and the benchmark portfolio weights.

For asset management firms who try to maximise expected relative return and minimise $T E_{S D}$, our study can provide solace for these firms whose expost $T E_{S D}$ becomes larger than the ex-ante $T E_{S D}$ the firms explained to their clients. Our results also suggest that if an investment technology firm presents models which claim to use ex-ante $T E_{S D}$ to accurately forecast expost $T E_{S D}$, one of the following two explanations may be true; the firm has included either a fudge factor or some rather sophisticated analysis based on the nature of the strategy that the fund will follow over the holding period. However, the latter method includes very difficult problems, and we feel that the former is more likely. Of course there is nothing wrong with "fudge" factors if they deliver the right answer, but typically clients would like to be told how the fudge operates.

This paper is organised as follows. In section 2, we first present definitions of $\mathrm{TE}$, and then in section 3 we show that when portfolio weights are stochastic, ex-ante $T E_{S D}$ is downward biased. Conclusions follow in section 4.

\section{Definitions of TE}

We introduce two different measures of TE to investigate ex-ante and expost differences in these measures. The first measure for TE is simply the standard deviation (or variance) of difference between portfolio returns and the benchmark portfolio returns, i.e., $T E_{S D}$. Roll (1992) analysed the relationship between the expected relative return and $T E_{S D}$, and showed that the locus of minimum $T E_{S D}$ portfolios for given expected relative return is located on the right of the global efficient frontier, unless the benchmark 
happens to be MV efficient. Thus, if the benchmark is global inefficient, the minimum $T E_{S D}$ portfolios will be inefficient.

Formally, let $\mathbf{r}_{\mathrm{t}}$ be a vector of rates of return at time $\mathrm{t}$ with mean vector ? and covariance matrix $\mathbf{S}$. Let the active portfolio weights at time $t$ be the vector $\mathbf{a}_{\mathrm{t}}$ and the benchmark weights be the vector $\mathbf{b}_{\mathrm{t}}$. Then

$$
\begin{aligned}
T E_{S D, t} & =\sqrt{\operatorname{var}\left(\mathbf{a}_{\mathrm{t}}^{\prime} \mathbf{r}_{\mathrm{t}}-\mathbf{b}_{\mathrm{t}}^{\prime} \mathbf{r}_{\mathrm{t}}\right)} \\
& =\sqrt{\left(\mathbf{a}_{\mathrm{t}}-\mathbf{b}_{\mathrm{t}}\right)^{\prime} \operatorname{var}\left(\mathbf{r}_{\mathrm{t}}\right)\left(\mathbf{a}_{\mathrm{t}}-\mathbf{b}_{\mathrm{t}}\right)} \\
& =\sqrt{\left(\mathbf{a}_{\mathrm{t}}-\mathbf{b}_{\mathrm{t}}\right)^{\prime} \Sigma\left(\mathbf{a}_{\mathrm{t}}-\mathbf{b}_{\mathrm{t}}\right) .}
\end{aligned}
$$

It is well understood that the portfolio weights, $\mathbf{w}_{\mathrm{t}}$, which is assumed to be non-stochastic ex-ante, will be stochastic ex-post. Since ex-post TE is computing TE from the actual portfolio returns, $r_{p t}$, where $r_{p t}=\mathbf{w}_{\mathrm{t}-1}^{\prime} \mathbf{r}_{\mathrm{t}}$, then a time series calculation of TE would involve, over a period from $\mathrm{t}=1, \ldots, \mathrm{T}$, the terms, $r_{p 1}, r_{p 2}, \ldots, r_{p T}$, or $\mathbf{w}_{0}^{\prime} \mathbf{r}_{1}, \mathbf{w}_{1}^{\prime} \mathbf{r}_{2}, \ldots, \mathbf{w}_{\mathrm{T}-1}^{\prime} \mathbf{r}_{\mathrm{T}}$. Conclusions about forecast failure arise from comparisons of ex-ante TE given by (1) versus the ex-post $\hat{T E} E_{S D}$ given by

$$
\hat{T E}_{S D}=\sqrt{\frac{1}{T-1} \sum_{t=1}^{T}\left(r_{p t}-\bar{r}_{p}\right)^{2}}
$$

Consider a fixed strategy such that at $\mathrm{t}=0$, we fix the weights $\mathbf{w}_{0}$. For randomness in $\mathbf{w}_{0}$ not to enter into the calculation, we would require that $\mathbf{w}_{\mathrm{t}}$ 1 would be rebased/rebalanced back to $\mathbf{w}_{0}$ within the time period from t- 1 to $\mathrm{t}$ and this would need to happen for all periods from $\mathrm{t}=0$ to $\mathrm{t}=\mathrm{T}-1$.

Barring the above case, all common strategies including passive strategies such as buy and hold, or "semi-active" ones such as quarterly re-balancing, tilting, etc., will involve $\mathbf{w}_{\mathrm{t}}$ being stochastic. The same will apply (obviously) to cap-weighted strategies.

Another definition of TE we use in this study is mean absolute deviations (MAD) of difference between portfolio returns and the benchmark portfolio returns, i.e., $T E_{M A D}$. Rudolf, Wolter and Zimmermann (1999) argued that the quadratic form of $T E_{S D}^{2}$ is difficult to interpret, and that 'portfolio managers typically think in terms of linear and not quadratic deviation from a benchmark'. $T E_{M A D}$ is defined as

$$
\hat{T E}_{M A D}=\frac{1}{T-1} \sum_{t=1}^{T}\left|r_{p t}-\bar{r}_{p}\right|
$$

Rudolf, Wolter and Zimmermann (1999), after comparing (2), (3), and some of their variants, argued that if performance fees of fund managers are 
linear, $T E_{M A D}$ describes investors' risk attitudes better than squared deviation. Most commercial packages, however, use $T E_{S D}$ in (2) rather than $T E_{M A D}$.

In this study our main concern is to investigate the effects of the stochastic nature of portfolio weights on the relationship between ex-ante and ex-post $T E_{S D}$ defined in (2). However, as in Rudolf, Wolter and Zimmermann (1999), if the performance fees of fund managers have a linear relationship with $T E_{M A D}$, it is also interesting to investigate the case.

\section{Active Management and Bias in Tracking Error}

It is our contention that the underestimation of TE comes from all portfolio construction, not just active management. In the conventional calculation, weights, $\mathbf{w}_{\mathrm{t}}$, are fixed at time $t$ and portfolio return $r_{p t+1}$ can be written as

$$
r_{p t+1}=\mathbf{w}_{t}^{\prime} \mathbf{r}_{t+1}
$$

so

$$
\begin{aligned}
\operatorname{var}\left(r_{p t+1}\right) & =\operatorname{var}\left(\mathbf{w}_{t}^{\prime} \mathbf{r}_{t+1}\right) \\
& =\mathbf{w}_{t}^{\prime} \mathbf{O} \mathbf{w}_{t}
\end{aligned}
$$

where $\mathbf{O}$ is the conditional (or unconditional) covariance matrix of $\mathbf{r}_{t+1}, \mathbf{w}_{t}$ being treated as fixed and $E\left(\mathrm{r}_{t+1}\right)=\boldsymbol{\mu}$ again being interpreted conditionally or unconditionally.

Let $\mathbf{e}$ be a vector of ones, i.e., $\mathbf{e}=\left(\begin{array}{lll}1 & 1 & \ldots\end{array}\right)^{\prime}$ '. Then, if we compute variances, then we need $\mathbf{e}^{\prime} \mathbf{w}_{t}=1$. However, if we compute $\mathrm{TE}$, then we have $\mathbf{e}^{\prime} \mathbf{w}_{t}=0$. Note that using the notation in equation (1), we can write $\mathbf{w}_{t}=\mathbf{a}_{t}-\mathbf{b}_{t}$. Otherwise the problems are the same.

We first propose Theorem 1 for the relationship between ex-post and exante $T E_{S D}^{2}$.

\section{Theorem 1}

If $\boldsymbol{w}_{t}$ that satisfies $\mathbf{e}^{\prime} \mathbf{w}_{t}=0$ is stochastic, i.e., $\mathbf{w}_{t}=\boldsymbol{\mu}_{w}+\boldsymbol{?}_{t}$, where $\mathbf{v}_{t} \sim\left(\mathbf{0}, \mathbf{O}_{w}\right)$, then the ex-post variance of the difference between portfolio returns and benchmark portfolio returns, $\hat{T E}_{S D}^{2}$, can be decomposed as follows;

$$
\hat{T E}_{S D}^{2}=\boldsymbol{\mu}^{\prime} \mathbf{O}_{w} \boldsymbol{\mu}+\operatorname{tr}\left(\mathbf{O O} \mathbf{O}_{w}\right)+\boldsymbol{\mu}_{w}^{\prime} \mathbf{O}_{w} .
$$

Proof. Since (4) is the population mean of the ex-post $\hat{T E}_{S D}$ given by (2), 


$$
\begin{aligned}
\hat{T E}_{S D}^{2} & =\operatorname{var}\left(r_{p t+1}\right) \\
& =\operatorname{var}\left(E\left(r_{p t+1} \mid \mathbf{w}_{t}\right)\right)+E\left(\operatorname{var}\left(r_{p t+1}\right) \mid \mathbf{w}_{t}\right) \\
& =\operatorname{var}\left(\mathbf{w}_{t}^{\prime} \boldsymbol{\mu}\right)+E\left(\mathbf{w}_{t}^{\prime} \mathbf{O} \mathbf{w}_{t}\right) \\
& =\operatorname{var}\left(\mathbf{w}_{t}^{\prime} \boldsymbol{\mu}\right)+E\left[\left(\boldsymbol{\mu}_{w}+\mathbf{v}_{t}\right)^{\prime} \mathbf{O}\left(\boldsymbol{\mu}_{w}+\mathbf{v}_{t}\right)\right] \\
& =\operatorname{var}\left(\mathbf{w}_{t}^{\prime} \boldsymbol{\mu}\right)+E\left[\mathbf{v}_{t}^{\prime} \mathbf{O} \mathbf{v}_{t}+2 \mathbf{v}_{t}^{\prime} \mathbf{O} \boldsymbol{\mu}_{w}+\boldsymbol{\mu}_{w}{ }^{\prime} \mathbf{O} \boldsymbol{\mu}_{w}\right] \\
& =\boldsymbol{\mu}^{\prime} \mathbf{O}_{w} \boldsymbol{\mu}+\operatorname{tr}\left(\mathbf{O O} \mathbf{O}_{w}\right)+\boldsymbol{\mu}_{w}^{\prime} \mathbf{O} \boldsymbol{\mu}_{w} \cdot Q E D .
\end{aligned}
$$

Note that the term $\operatorname{tr}\left(\mathbf{O O} \mathbf{O}_{w}\right)$ is positive since it can be interpreted as the expectation of $\mathbf{v}_{t}{ }^{\prime} \mathbf{O}_{w} \mathbf{v}_{t}$ which expectation will be positive with probability 1. In addition, the positivity of $\boldsymbol{\mu}^{\prime} \mathbf{O}_{w} \boldsymbol{\mu}$ follows from the positive definiteness of $\mathbf{O}_{w}$. Since all three terms in the above equation are nonnegative, the variance of the portfolio is higher than the portfolio variance taken at the average portfolio weight $\boldsymbol{\mu}_{w}^{\prime} \mathbf{O} \boldsymbol{\mu}_{w}$.

Remark 1. In the case of non-stochastic weights $\mathbf{w}_{t}=\boldsymbol{\mu}_{w}, \mathbf{O}_{w}=0$, and $\operatorname{var}\left(r_{p t+1}\right)=\boldsymbol{\mu}_{w}^{\prime} \mathbf{O} \boldsymbol{\mu}_{w}$. This is the ex-post tracking error with fixed weights.

Remark 2. It also follows that since $\mathbf{e}^{\prime} \mathbf{w}_{t}=0$ for all $t, \mathbf{e}^{\prime} \boldsymbol{\mu}_{w}=0$ and $\mathbf{e}^{\prime} \mathbf{O}_{w} \mathbf{e}=0$. Thus if there is little variation in $\mathrm{m}$ so that $\mathrm{m}$ is nearly collinear with $\boldsymbol{e}$, the term $\boldsymbol{\mu}^{\prime} \mathbf{O}_{w} \boldsymbol{\mu}$ should be very nearly zero. Thus, if there is little variability in $m$ over the period that the fund is being measured, we would expect most of the bias from $\boldsymbol{\mu}_{w}^{\prime} \mathbf{O} \boldsymbol{\mu}_{w}$. Lawton-Browne (2000) establishes that $\boldsymbol{\mu}^{\prime} \mathbf{O}_{w} \boldsymbol{\mu}$ is very small in the cases she examines.

This result establishes that calculations based on treating portfolio weights as fixed must underestimate the ex-post tracking error over a historical period if the weights are not kept fixed. So if we take a particular fund, compute its monthly rate of return, $r_{p t}$, and then calculate the tracking error/variance over a period $T(t=1, T)$, where the weights have not been rebalanced monthly prior to reporting the returns, we should expect underestimation of the actual tracking error.

Having identified the disease, finding the cure seems to be rather hard. If, over the period being analysed, we store the weights $\left(\mathbf{w}_{t}, t=1, \ldots, T\right)$, we can estimate $\boldsymbol{\mu}_{w}$ and $\mathbf{O}_{w}, \hat{\boldsymbol{\mu}}_{w}=\sum_{t=1}^{T} \mathbf{w}_{t} / T$ and $\hat{\mathbf{O}}_{w}=\frac{1}{T} \sum_{t=1}^{T} \mathbf{w}_{t} \mathbf{w}_{t}^{\prime}-\hat{\boldsymbol{\mu}}_{w} \hat{\boldsymbol{\mu}}_{w}^{\prime}$. Armed with these estimates we can get a much more accurate measure of $T E_{S D}$ but the analysis is ex-post. Ex-ante analysis would require assessing 
the type of strategy the manager wishes to indulge in and converting these strategies into parameter estimates so that we might expect quarterly rebalancing to result in errors of a certain magnitude, for example. Such strategy-based estimates could be calculated and would be a useful research contribution.

More simple minded solutions are already in existence. Planned Sponsors often require that managers limit their turnover or specific exposures. Although these requirements are usually motivated by considerations of transaction costs or concerns of risk/bankruptcies of specific companies, they can also be interpreted as pragmatic ways of reducing $\mathbf{O}_{w}$.

In the above, it might be thought that randomness in the weights might move them so as to reduce the tracking error. In tracking error problems $\mathbf{e}^{\prime} \mathbf{w}_{t}=0$ and so there may be stochastic realisations of $\mathbf{w}_{\mathrm{t}}$ that make all the weights zero in which case the tracking error is of course reduced to zero. In the above calculations, it is assumed that $\operatorname{prob}\left(w_{j t}=0, j=1, n\right)$ is zero. That is, mathematically, we exclude the possibility that $\mathbf{w}_{t}$ is the zero vector. In practical terms we assume that you will not hold the benchmark each period.

If the benchmark is cap-weighted, as most are, then over the holding period, its weights will change. Even if the fund being measured just does buy and hold or quarterly rebalancing, there will be a random pattern in the overall weights because the cap-weight of the benchmark changes over time.

This is recognised by Gardner and Bowie (2000) who, in their section 3.1, distinguish between experienced versus prospective tracking errors. The former is based on the returns of the portfolio versus returns of the benchmark taken over the holding period. The latter takes initial weights, $\mathbf{w}_{t}$, and computes, ex-ante, an estimate of $\mathbf{O}$, then the ex-ante $T E_{S D}^{2}$ is $\mathbf{w}_{t}^{\prime} \mathbf{O} \mathbf{w}_{t}$.

It would be our contention that both are informative ex-post. That is using the historical return data over the holding period, and possibly data predated that period, we could compute both measures. By subtracting (4) from (5) we get an estimate of $\boldsymbol{\mu}^{\prime} \mathbf{O}_{w} \boldsymbol{\mu}+\operatorname{tr}\left(\mathbf{O O} \mathbf{O}_{w}\right)$ if $\boldsymbol{\mu}_{w}=\mathbf{w}_{t}$.

If we can convince ourselves that $\mathbf{O}_{\mathrm{w}}=0$, then a comparison of $\mathbf{w}_{t}^{\prime} \mathbf{O} \mathbf{w}_{t}$ pre- and post- sample would allow us to see the impact of mis-estimation of individual asset risk. 
We cannot apply the same argument as in Theorem 1 to the relationship between ex-post and ex-ante $T E_{M A D}$ 's For the MAD case, we propose Theorem 2.

\section{Theorem 2}

If $\mathbf{w}_{\mathrm{t}}$ that satisfies $\mathbf{e}^{\prime} \mathbf{w}_{t}=0$ is stochastic, i.e., $\mathbf{w}_{t}=\boldsymbol{\mu}_{w}+\boldsymbol{?}_{t}$, where $\mathbf{v}_{t} \sim\left(\mathbf{0}, \mathbf{O}_{w}\right)$, then the ex-post mean absolute deviations of the difference between portfolio returns and benchmark portfolio returns, $\hat{T E}_{M A D}$, can be shown as

$$
\hat{T E}_{M A D} \leq\left|\boldsymbol{\mu}_{w}^{\prime} \boldsymbol{\mu}\right|+E\left(\left|\mathbf{v}_{t}^{\prime} \mathbf{r}_{t}\right|\right) .
$$

Proof. Using the law of iterated expectations, we have

$$
\begin{aligned}
\hat{T E_{M A D}} & =E_{w_{t}}\left[E\left(\mid r_{p t} \| \mathbf{w}_{t}\right)\right] \\
& =E_{w_{t}}\left[E\left(\mid\left(\boldsymbol{\mu}_{w}+\mathbf{v}_{t}\right)^{\prime} \mathbf{r}_{t} \| \mathbf{w}_{t}\right)\right] \\
& =E_{w_{t}}\left[E\left(\mid \boldsymbol{\mu}_{w}^{\prime} \mathbf{r}_{t}+\mathbf{v}_{t}^{\prime} \mathbf{r}_{t} \| \mathbf{w}_{t}\right)\right] \\
& \leq E_{w_{t}}\left[\left|\boldsymbol{\mu}_{w}^{\prime} \boldsymbol{\mu}\right|+E\left(\mid \mathbf{v}_{t}^{\prime} \mathbf{r}_{t} \| \mathbf{v}_{t}\right)\right] \\
& =\left|\boldsymbol{\mu}_{w}^{\prime} \boldsymbol{\mu}\right|+E_{v_{t}}\left[E\left(\mid \mathbf{v}_{t}^{\prime} \mathbf{r}_{t} \| \mathbf{v}_{t}\right)\right] \\
& =\left|\boldsymbol{\mu}_{w}^{\prime} \boldsymbol{\mu}\right|+E\left(\left|\mathbf{v}_{t}^{\prime} \mathbf{r}_{t}\right|\right) . Q E D .
\end{aligned}
$$

Note that $\left|\boldsymbol{\mu}_{w} \boldsymbol{\mu}\right|$ can be interpreted as the ex-ante $T E_{M A D}$. If the weight, $\mathbf{w}_{\mathrm{t}}$, is not stochastic, then $\mathbf{w}_{t}=\boldsymbol{\mu}_{w}$ and $\hat{T E_{M A D}}=\left|\boldsymbol{\mu}_{w}{ }_{w}^{\prime} \boldsymbol{\mu}\right|$. That is, ex-post and exante $T E_{M A D}$ 's are same. In addition, we have the following two remarks for $T E_{M A D}$.

Remark 3. It follows that if $\mathbf{r}_{\mathrm{t}}$ is not correlated with $\boldsymbol{v}_{t}$, then for many stocks the term $E\left(\left|\mathbf{v}_{t} \mathbf{r}_{t}\right|\right)$ may be very close to zero. Thus, in this case, we can obtain $\hat{T E}_{M A D} \leq\left|\boldsymbol{\mu}_{w}^{\prime} \boldsymbol{\mu}\right|$.

Remark 4. If $E\left(\left|\mathbf{v}_{t}^{\prime} \mathbf{r}_{t}\right|\right)$ is not negligible, we cannot decide if ex-post $T E_{M A D}$ is larger than ex-ante $T E_{M A D}$; that is, $\hat{T E_{M A D}} \stackrel{>}{=}\left|\boldsymbol{\mu}_{w}^{\prime} \boldsymbol{\mu}\right|$.

Remarks 3 and 4 show that we are unable to prove results similar to Theorem 1. In fact, Remark 3 suggests that ex-post $T E_{M A D}$ may be smaller than ex-ante $T E_{M A D}$ when $E\left(\left|\mathbf{v}_{t} \mathbf{r}_{t}\right|\right)$ is close to zero. 
As in the case of $T E_{S D}$, the magnitude of the components in (7) are not known and thus we can not conclude if calculations with fixed portfolio weights underestimate the ex-post tracking error over a historical period if the weights are stochastic.

\section{Conclusions}

We have observed cases when TE becomes influential in the investment management market. For example, sponsors of defined benefit plans increasingly pay attention to 'risk budgeting' that represents allocating TE across managers of different asset classes; see Gupta, Prajogi, and Stubbs (1999). Another example is that last year Barclays Global Investors Ltd agreed to return back a portion of its management fee to plan sponsor $\mathbf{J}$. Sainsbury PLC Pension Scheme, if the firm exceeded its agreed TE limits. ${ }^{2}$

If TE is used to measure the performance of active funds as in the cases above, the bias we find in this study should be considered. That is, fund managers should allow bias when they begin to make portfolio strategy; the planned TE should be less than the target TE because of the bias.

Unfortunate, the magnitude of the TE bias is not known to the authors. In this issue, Lawton-Browne (2000) presents results which suggest that it will more or less double ex-ante TE measured on an annual basis. Other calculations the authors have seen with different packages produce much less bias. Thus there appears to be some variation in different investment technologies bias production. However, in cases where there appears to be evidence of bias-correction, no methodology of bias-correction is explained.

\section{References}

Baierl, G. T. and P. Chen, 2000, "Choosing Managers and Funds," Journal of Portfolio Management 26(2), 47-53.

Gardner, D. and D. Bowie, 2000, M Brooks and M. Cumberworth, "Predicted Tracking Errors Fact or Fantasy?," Faculty and Institute of Actuaries, Investment Conference paper, 25-27 June 2000.

Grinold, R. and R. Kahn, 1995, Active Portfolio Management, Irwin.

Gupta, F., R. Prajogi, and E. Stubbs, 1999, "The Information Ratio and Performance," Journal of Portfolio Management (Fall 1999), 33-39.

217 April 2000, Pension \& Investments, Volume 28, Issue 8, Page 18. 
Larsen, G. A. and B. G. Resnick,, 1998, "Empirical Insights on Indexing," Journal of Portfolio Management 25(1), 51-60.

Lawton-Browne, C. L. 2001, Cross-reference, Journal of Asset Management.

Markowitz, H. M., 1959, Portfolio Selection, $1^{\text {st }}$ Edition, New York: John Wiley \& Sons.

Pope, P. and P. K. Yadav, 1994, "Discovering Error in Tracking Error," Journal of Portfolio Management (Winter 1994), 27-32.

Roll, R., 1992, "A Mean/Variance Analysis of Tracking Error," Journal of Portfolio Management, 13-22.

Rudolf, M., H. Wolter, and H. Zimmermann, 1999, "A Linear Model for Tracking Error Minimization," Journal of Banking and Finance 23, 85-103.

Sharpe, W, 1964, "Capital asset prices : A Theory of Capital Market Equilibrium under Conditions of Risk," Journal of Finance 19, 425442. 


\section{List of other working papers:}

\section{1}

1. Soosung Hwang and Steve Satchell, GARCH Model with Cross-sectional Volatility; GARCHX Models, WP01-16

2. Soosung Hwang and Steve Satchell, Tracking Error: Ex-Ante versus Ex-Post Measures, WP01-15

3. Soosung Hwang and Steve Satchell, The Asset Allocation Decision in a Loss Aversion World, WP01-14

4. Soosung Hwang and Mark Salmon, An Analysis of Performance Measures Using Copulae, WP01-13

5. Soosung Hwang and Mark Salmon, A New Measure of Herding and Empirical Evidence, WP01-12

6. Richard Lewin and Steve Satchell, The Derivation of New Model of Equity Duration, WP0111

7. Massimiliano Marcellino and Mark Salmon, Robust Decision Theory and the Lucas Critique, WP01-10

8. Jerry Coakley, Ana-Maria Fuertes and Maria-Teresa Perez, Numerical Issues in Threshold Autoregressive Modelling of Time Series, WP01-09

9. Jerry Coakley, Ana-Maria Fuertes and Ron Smith, Small Sample Properties of Panel Timeseries Estimators with I(1) Errors, WP01-08

10. Jerry Coakley and Ana-Maria Fuertes, The Felsdtein-Horioka Puzzle is Not as Bad as You Think, WP01-07

11. Jerry Coakley and Ana-Maria Fuertes, Rethinking the Forward Premium Puzzle in a Nonlinear Framework, WP01-06

12. George Christodoulakis, Co-Volatility and Correlation Clustering: A Multivariate Correlated ARCH Framework, WP01-05

13. Frank Critchley, Paul Marriott and Mark Salmon, On Preferred Point Geometry in Statistics, WP01-04

14. Eric Bouyé and Nicolas Gaussel and Mark Salmon, Investigating Dynamic Dependence Using Copulae, WP01-03

15. Eric Bouyé, Multivariate Extremes at Work for Portfolio Risk Measurement, WP01-02

16. Erick Bouyé, Vado Durrleman, Ashkan Nikeghbali, Gael Riboulet and Thierry Roncalli, Copulas: an Open Field for Risk Management, WP01-01

\section{0}

1. Soosung Hwang and Steve Satchell, Valuing Information Using Utility Functions, WP00-06

2. Soosung Hwang, Properties of Cross-sectional Volatility, WP00-05

3. Soosung Hwang and Steve Satchell, Calculating the Miss-specification in Beta from Using a Proxy for the Market Portfolio, WP00-04

4. Laun Middleton and Stephen Satchell, Deriving the APT when the Number of Factors is Unknown, WP00-03

5. George A. Christodoulakis and Steve Satchell, Evolving Systems of Financial Returns: AutoRegressive Conditional Beta, WP00-02

6. Christian S. Pedersen and Stephen Satchell, Evaluating the Performance of Nearest Neighbour Algorithms when Forecasting US Industry Returns, WP00-01

1999

1. Yin-Wong Cheung, Menzie Chinn and Ian Marsh, How do UK-Based Foreign Exchange Dealers Think Their Market Operates?, WP99-21 
2. Soosung Hwang, John Knight and Stephen Satchell, Forecasting Volatility using LINEX Loss Functions, WP99-20

3. Soosung Hwang and Steve Satchell, Improved Testing for the Efficiency of Asset Pricing Theories in Linear Factor Models, WP99-19

4. Soosung Hwang and Stephen Satchell, The Disappearance of Style in the US Equity Market, WP99-18

5. Soosung Hwang and Stephen Satchell, Modelling Emerging Market Risk Premia Using Higher Moments, WP99-17

6. Soosung Hwang and Stephen Satchell, Market Risk and the Concept of Fundamental Volatility: Measuring Volatility Across Asset and Derivative Markets and Testing for the Impact of Derivatives Markets on Financial Markets, WP99-16

7. Soosung Hwang, The Effects of Systematic Sampling and Temporal Aggregation on Discrete Time Long Memory Processes and their Finite Sample Properties, WP99-15

8. Ronald MacDonald and Ian Marsh, Currency Spillovers and Tri-Polarity: a Simultaneous Model of the US Dollar, German Mark and Japanese Yen, WP99-14

9. Robert Hillman, Forecasting Inflation with a Non-linear Output Gap Model, WP99-13

10. Robert Hillman and Mark Salmon , From Market Micro-structure to Macro Fundamentals: is there Predictability in the Dollar-Deutsche Mark Exchange Rate?, WP99-12

11. Renzo Avesani, Giampiero Gallo and Mark Salmon, On the Evolution of Credibility and Flexible Exchange Rate Target Zones, WP99-11

12. Paul Marriott and Mark Salmon, An Introduction to Differential Geometry in Econometrics, WP99-10

13. Mark Dixon, Anthony Ledford and Paul Marriott, Finite Sample Inference for Extreme Value Distributions, WP99-09

14. Ian Marsh and David Power, A Panel-Based Investigation into the Relationship Between Stock Prices and Dividends, WP99-08

15. Ian Marsh, An Analysis of the Performance of European Foreign Exchange Forecasters, WP99-07

16. Frank Critchley, Paul Marriott and Mark Salmon, An Elementary Account of Amari's Expected Geometry, WP99-06

17. Demos Tambakis and Anne-Sophie Van Royen, Bootstrap Predictability of Daily Exchange Rates in ARMA Models, WP99-05

18. Christopher Neely and Paul Weller, Technical Analysis and Central Bank Intervention, WP9904

19. Christopher Neely and Paul Weller, Predictability in International Asset Returns: A Reexamination, WP99-03

20. Christopher Neely and Paul Weller, Intraday Technical Trading in the Foreign Exchange Market, WP99-02

21. Anthony Hall, Soosung Hwang and Stephen Satchell, Using Bayesian Variable Selection Methods to Choose Style Factors in Global Stock Return Models, WP99-01

\section{8}

1. Soosung Hwang and Stephen Satchell, Implied Volatility Forecasting: A Compaison of Different Procedures Including Fractionally Integrated Models with Applications to UK Equity Options, WP98-05

2. Roy Batchelor and David Peel, Rationality Testing under Asymmetric Loss, WP98-04

3. Roy Batchelor, Forecasting T-Bill Yields: Accuracy versus Profitability, WP98-03

4. Adam Kurpiel and Thierry Roncalli, Option Hedging with Stochastic Volatility, WP98-02

5. Adam Kurpiel and Thierry Roncalli, Hopscotch Methods for Two State Financial Models, WP98-01 